\title{
Relación entre hipotiroidismo y síndrome de ovario poliquístico
}

\section{The relationship between hypothyroidism and polycystic ovary syndrome}

\section{Julieth A. Guzmán-López ${ }^{\dagger *}$ Oscar E. Rivera-Contreras y y Janer Sepúlveda-Agudelo²}

Escuela de Medicina, Facultad de Salud, Universidad Industrial de Santander; ${ }^{2}$ Departamento de Ginecobstetricia, Facultad de Salud, Universidad Industrial de Santander. Bucaramanga, Santander, Colombia

\section{Resumen}

Introducción: Se ha documentado la asociación del síndrome de ovario poliquístico con alteraciones metabólicas y enfermedades cardiovasculares. Su relación con trastornos autoinmunitarios no está claramente establecida, pero se ha encontrado una importante prevalencia de desórdenes tiroideos en pacientes con síndrome de ovario poliquístico. Objetivo: Describir las diferentes teorías existentes que puedan explicar la relación entre hipotiroidismo y síndrome de ovario poliquístico, junto con su posible impacto en la morbilidad asociada. Método: Se realizó una búsqueda en PubMed y LILACS con las palabras clave "Polycystic ovary síndrome","Hypotyroidism,"“thyroid disease” y sus respectivos términos en español, durante julio de 2020. Resultados: Se seleccionaron 51 artículos relacionados con el tema, publicados en los últimos 10 años. La fisiopatogenia entre ambos trastornos no está claramente establecida. Se ha encontrado un importante impacto metabólico en este grupo de pacientes y se considera que su riesgo cardiovascular podría estar aumentado. Conclusiones: Al considerarse la prevalencia significativa y las complicaciones que tanto a corto como a largo plazo podrían tener las mujeres con ambas alteraciones, se hace necesario discutir la necesidad de la exclusión del hipotiroidismo de los criterios diagnósticos aplicados para el síndrome, la tamización temprana y el estudio de las implicaciones terapéuticas que trae su abordaje.

Palabras clave: Hipotiroidismo. Síndrome de ovario poliquístico. Fisiopatología.

\section{Abstract}

Introduction: The association of polycystic ovary syndrome with other metabolic disorders and cardiovascular diseases has been documented; nevertheless, its relationship with autoimmune disorders is not clearly established, however, an important prevalence of thyroid disorders has been found in this group of patients. Objective: To describe the different existing theories that can explain the relationship between hypothyroidism and polycystic ovary syndrome along with its possible impact on associated morbidities. Method: A search was conducted in PubMed and LILACS with the keywords of "Polycystic ovary syndrome","'Hypothyroidism,"“Thyroid disease” and with its respective Spanish terms, in July 2020. Results: 51 articles related to the subject were selected, published in the last 10 years. The pathogenesis between both disorders is not clearly established. An important impact has been found at the metabolic level in this group of patients and it is considered that their cardiovascular risk could be increased. Conclusions: Considering the significant prevalence and complications that both short and long term, women with both alterations could have, it is necessary to discuss the need for the exclusion of hypothyroidism from the diagnostic criteria applied for the syndrome, early screening of the syndrome, and the study of the therapeutic implications that its approach brings.

Key words: Hypothyroidism. Polycystic ovary syndrome. Pathophysiology.

\section{Correspondencia:}

*Julieth A. Guzmán-López

E-mail: jalexandraguzman@hotmail.com
Disponible en internet: 23-12-2021
Fecha de recepción: 27-11-2020 Fecha de aceptación: 08-11-2021 DOI: $10.24875 /$ RECHOG.M2100003
Rev Chil Obstet Ginecol. 2021;86(5):485-491
www.rechog.com

Rev Chil Obstet Ginecol. 2021;86(5):485-491
www.rechog.com ste es un artículo open access bajo la licencia 


\section{Introducción}

El síndrome de ovario poliquístico (SOP) es el trastorno endocrinológico más frecuente en las mujeres en edad reproductiva'. Tiene una amplia presentación clínica, y ciertos factores ambientales, genéticos y metabólicos tienen un papel importante ${ }^{2}$. La prevalencia de este trastorno varía del $6 \%$ al $21 \%$, dependiendo de la población y de los criterios utilizados para su diagnóstico ${ }^{2,3}$.

Actualmente, el correcto diagnóstico de SOP se realiza con los criterios de Rotterdam de 2003: hiperandrogenismo clínico o bioquímico, presencia de ovarios poliquísticos por ultrasonografía y disfunción ovárica, típicamente definida como ciclos menstruales $\geq 350$ $\leq 21$ días; el cumplimiento de al menos dos de dichos criterios es diagnóstico para el síndrome ${ }^{4}$. A partir de esto, se establecieron cuatro fenotipos resultantes: el $A$ y el $\mathrm{B}$, conocidos como «clásicos», cuya sintomatología y riesgo de complicaciones metabólicas son mayores, y el $C$ y el $D$, que se caracterizan por tener una menor prevalencia de disfunción endocrina (Tabla 1)5. Para el diagnóstico del trastorno se deben excluir otras patologías que cursen con manifestaciones clínicas semejantes, tales como el hipotiroidismo. Sin embargo, se ha encontrado en diferentes estudios una alta prevalencia de enfermedad tiroidea en mujeres con SOP, registrándose una frecuencia de tiroiditis de Hashimoto y de hipotiroidismo subclínico de hasta un $27 \%$ y un $11,3-$ $22.5 \%$, respectivamente ${ }^{6-10}$. De igual forma, se ha visto un incremento, estadísticamente significativo, de hormona estimulante de la tiroides (TSH), anticuerpos antiperoxidasa, anticuerpos antitiroglobulina y presencia de bocio en pacientes con SOP en comparación con pacientes control ${ }^{11}$. Ante la aclaración sobre la necesidad de exclusión del hipotiroidismo en mujeres con SOP y la posible relación entre estas dos patologías, se realizó esta revisión con el objetivo de describir las diferentes teorías existentes que podrían explicar dicha relación y su posible impacto en las pacientes.

\section{Método}

Se realizó una búsqueda bibliográfica en las bases de datos de PubMed y LILACS, durante julio de 2020, usando los términos MeSH "Polycystic ovary syndrome" y "Hypotyroidism, thyroid disease", y los términos DeCS "Síndrome de ovario poliquístico, hipotiroidismo, asociación". Se buscaron estudios observacionales, analíticos, artículos de revisión, capítulos de libro, revisiones sistemáticas y metaanálisis. Los
Tabla 1. Fenotipos del síndrome de ovario poliquístico (Rotterdam 2003)

\begin{tabular}{|l|l|l|l}
\hline Fenotipo & Hiperandrogenismo & $\begin{array}{l}\text { Disfunción } \\
\text { ovárica }\end{array}$ & $\begin{array}{l}\text { Ovario } \\
\text { poliquístico } \\
\text { ecográfico }\end{array}$ \\
\hline A & X & X & X \\
B & X & X & \\
C & X & & $X$ \\
D & & $X$ & $X$ \\
\hline
\end{tabular}

criterios de inclusión fueron que los artículos trataran sobre el SOP y su asociación con el hipotiroidismo, estar escritos en español o inglés, y haber sido publicados en los últimos 10 años. En la búsqueda se obtuvieron 395 artículos, obteniendo 51 artículos únicos, y finalmente se incluyeron 15 artículos adicionales, encontrados en las referencias de los textos incluidos, que cumplieron con los criterios previamente establecidos.

\section{Resultados}

\section{Patogenia}

La fisiopatología que asocia el hipotiroidismo y el SOP aún no ha sido claramente establecida ${ }^{12}$; sin embargo, en la actualidad existen diferentes planteamientos que explican esta posible relación y que a continuación se exponen.

\section{GenÉTICO}

A partir de resultados de un estudio en gemelas, se sugiere que la patogénesis del SOP tiene una importante influencia genética, por encima del $70 \%{ }^{13}$. En trabajos latinoamericanos en familiares de primer grado de mujeres con SOP se ha diagnosticado el síndrome en alrededor del $38 \%$ de los casos $^{14}$; de igual forma, se ha estimado una prevalencia para madres y hermanas de pacientes con SOP del $8-24 \%$ y el $16-22 \%$, respectivamente ${ }^{15,16}$. En cuanto al hipotiroidismo, la presencia de tiroiditis de Hashimoto se ha reportado en el $17 \%$ de familiares de primer grado de pacientes con la enfermedad, encontrándose un riesgo nueve veces más alto de desarrollar la patología en comparación con la población general ${ }^{17}$.

Actualmente se afirma la existencia de genes en común, que al estar mutados podrían explicar la 
presencia de ambas patologías. Entre estos se encuentra el gen de la fibrilina 3 (FBN3) ${ }^{18,19}$, clave en la función del factor de crecimiento transformante beta (TGF $\beta$ ), que tiene un importante papel en la actividad de las células $T$ reguladoras (Treg), implicadas en la regulación de la respuesta autoinmunitaria, encontrándose que bajos niveles de TGF $\beta$ y de Treg podrían estar relacionados con la aparición de tiroiditis de Hashimoto ${ }^{18,19}$. Adicionalmente, se ha reportado que el locus D19S884 del alelo 8 en el gen FBN3 en mujeres con SOP y tiroiditis de Hashimoto conlleva niveles bajos de TGF $\beta^{19}$. Por otro lado, se ha involucrado en este tipo de trastornos al gen CYP1B1, encargado de codificar una enzima para la oxidación del 17b-estradiol a 4-hidroxiestradiol, a causa de que su polimorfismo ha sido relacionado con cambios en los niveles de tiroxina (T4), de fracción libre de tiroxina (FT4) y de fracción libre de triyodotironina (FT3) en mujeres con $\mathrm{SOP}^{20}$. De igual manera, la variante del receptor de la hormona liberadora de gonadotropina rs1038426 ha sido relacionada con cambios significativos en la secreción de insulina, la resistencia a la insulina y los niveles de $\mathrm{TSH}^{21}$.

\section{HORMONAS SEXUALES}

En mujeres con SOP se presenta un importante aumento de la hormona luteinizante, incluso en la fase folicular del ciclo menstrual ${ }^{22,23}$, lo que aunado a la similitud estructural y funcional con la TSH permite que tenga efectos tirotrópicos que llevan a un aumento del volumen tiroideo ${ }^{23}$. Asimismo, se ha afirmado que la alta prevalencia de tiroiditis de Hashimoto en este grupo de mujeres podría deberse al papel del desbalance entre estrógenos y progesterona en el aumento de la inmunidad en estas pacientes ${ }^{24}$. Lo anterior se explica porque, ante el incremento de los estrógenos, se estimulan más fácilmente los linfocitos $B$ y se evita la inhibición de las células T supresoras, promoviendo la formación de anticuerpos a través de la respuesta inmunitaria de linfocitos Th2 ${ }^{24}$. Además, se conoce que el papel atenuante de los andrógenos sobre las enfermedades autoinmunitarias es insuficiente en las mujeres con SOP, lo que lleva al desarrollo de trastornos inmunitarios ${ }^{6,11,24}$. Asimismo, se considera que la presencia de tiroiditis de Hashimoto sería más frecuente en el fenotipo sin hiperandrogenemia, seguido por los clásicos y el subtipo $\mathrm{C}^{19}$.

\section{OBESIDAD Y RESISTENCIA A LA INSULINA}

La obesidad en las pacientes con SOP es significativamente más alta que en aquellas sin este síndrome, reportándose una prevalencia del $42 \%$ al $80 \%{ }^{25,26}$. La resistencia a la insulina es frecuente encontrarla, en el $60-70 \%$ de los $\operatorname{casos}^{27}$. Se ha establecido la asociación entre la obesidad y la presencia de hipotiroidismo subclínico ${ }^{28}$, siendo esta explicada por dos vías principales: la primera se asocia a la condición proinflamatoria propia de la obesidad, lo que lleva a una disminución de la deiodinasa 2 presente en la hipófisis, incrementando los niveles de $\mathrm{TSH}^{29}$; y la segunda hace alusión al papel de la leptina sobre el hipotálamo en la producción de hormona liberadora de tirotropina (TRH) y la mediación que puede tener en la autoinmunidad al estimular los linfocitos $T$ efectores $e$ inhibir los linfocitos $T$ reguladores, explicando la asociación entre al aumento de la leptina en pacientes con incremento de adiposidad visceral y trastornos tiroideos autoinmunitarios ${ }^{30,31}$. Sin embargo, a pesar de lo anterior, es escasa la información publicada referente a la relación entre la obesidad en mujeres con SOP y la alteración en la función tiroidea. En el único estudio publicado sobre el tema, no se encontraron diferencias estadísticamente significativas entre el aumento de la TSH en mujeres con SOP y exceso de peso en comparación con mujeres delgadas ${ }^{32}$.

Contrario a la obesidad, la resistencia a la insulina no ha demostrado tener asociación estadísticamente significativa con la presencia de hipotiroidismo ${ }^{28}$; no obstante, se ha encontrado una fuerte asociación con el incremento del volumen tiroideo ${ }^{33}$. Esto se explicaría por el efecto estimulante de la hiperinsulinemia resultante en la proliferación y la hiperplasia celular, junto con la inhibición de los procesos apoptóticos ${ }^{34}$. Adicionalmente, aunque se han realizado estudios que afirman una disminución significativa de la TSH en pacientes con hipotiroidismo y SOP ante la administración de metformina, sola o combinada con otros medicamentos como el inositol, no hay suficiente evidencia para demostrar que se deba a la reducción de la resistencia a la insulina, sino más bien a otras posibles hipótesis, como el cambio en la afinidad o en el número de receptores, el incremento en el tono central dopaminérgico, e incluso el efecto directo que pueda tener la metformina en la regulación de la $\mathrm{TSH}^{35,36}$.

\section{Vitamina D}

Se ha considerado que la deficiencia de vitamina $D$ es un factor de riesgo para el desarrollo de enfermedades autoinmunitarias, tales como la diabetes mellitus, la esclerosis múltiple, la enfermedad inflamatoria intestinal, el lupus eritematoso sistémico, la artritis reumatoidea y 
las enfermedades tiroideas autoinmunitarias ${ }^{37,38}$. Lo anterior se debe al papel de su forma activa en la modulación del sistema inmunitario, ya que a través de receptores de la vitamina D (VDR) y la enzima 1 $\alpha$-hidroxilasa, importante en la síntesis de calcitriol, influye en el incremento de las células Treg, así como en la inhibición de la diferenciación y proliferación de las células Th1, la producción de citocinas (interleucina [IL] 2, interferón gamma, IL-17 e IL-21) ${ }^{39}$ y la estimulación de las células Th2 en la producción de citocinas antiinflamatorias (IL-3, IL-4, IL-5, e IL-10) y las natural killer. Por otro lado, se ha reportado que la vitamina $D$ cumple funciones en la inducción apoptótica y la inhibición de la proliferación de las células $\mathrm{B}$ y su diferenciación en células plasmáticas, como también en el bloqueo de la secreción de inmunoglobulinas y la generación de células $\mathrm{B}$ de memoria ${ }^{39}$. Por lo tanto, se ha valorado esta capacidad de la vitamina D para suprimir la respuesta inmunitaria adaptativa como un posible efecto protector para diversas patologías de componente autoinmunitario, al describirse el potencial papel de su suplementación en la prevención y la reducción de la gravedad y la actividad de la enfermedad $^{40}$. Sin embargo, esta evidencia no ha sido considerada suficiente para establecer una relación clara entre la deficiencia de vitamina $D$ y la presentación de este tipo de patologías, por lo que se ha recomendado la realización de ensayos clínicos aleatorizados para evaluar la causalidad entre estas dos condiciones ${ }^{40}$.

Al igual que lo anterior, en diferentes estudios se ha observado una relación entre bajos niveles de vitamina D y trastornos tiroideos, al encontrarse resultados estadísticamente significativos en pacientes con deficiencia de vitamina $D$ y presencia de alteraciones autoinmunitarias como la enfermedad de Graves y la tiroiditis de Hashimoto ${ }^{41,42}$. Aunque existen resultados heterogéneos sobre la deficiencia de vitamina $D$ en mujeres con SOP, se ha demostrado que valores bajos de esta vitamina son mucho más frecuentes en las mujeres con SOP que en los grupos control, haciendo concluir la posible existencia de una relación entre la deficiencia de vitamina $D$ y la gravedad del SOP ${ }^{43,44}$. Hasta el momento, solo en un estudio, con varias limitaciones, se evaluaron los niveles de 25-hidroxi vitamina $D$ en relación con la presencia de enfermedades tiroideas autoinmunitarias en pacientes con SOP, encontrando unos valores significativamente más bajos en mujeres con ambas patologías ${ }^{45}$.

\section{TIMO}

Esta glándula tiene un importante papel en la modulación del sistema inmunitario, al prevenir la autoinmunidad a través de la eliminación de las células T autorreactivas durante el periodo fetal, así como por el efecto supresivo de las células Treg ${ }^{19,46}$. Se ha planteado, a partir de estudios en roedores, que valores elevados de estrógenos antes de los 10 días de gestación pueden afectar a la maduración del timo, y con ello la producción de Treg y la predisposición a ovarios poliquísticos ${ }^{47}$. Del mismo modo, en modelos animales se ha observado que los esteroides adrenales, como la corticosterona, pueden ser causa de una disminución de timocitos y del peso del timo, al igual que desencadenantes de anovulación y desarrollo de quistes ováricos $^{48}$. En suma, las alteraciones del timo en el periodo prenatal, ya sea por estrógenos o por esteroides adrenales, podrían estar involucradas en la presentación de SOP en mujeres predispuestas. No obstante, esto no ha sido probado en la coexistencia con tiroiditis de Hashimoto, sino más bien por mecanismos separados ${ }^{19}$, encontrándose que bajos niveles de Treg podrían ser un mecanismo clave en mujeres con tiroiditis de Hashimoto, dada su etiología autoinmunitaria, mientras que en el SOP sería la estrogenización del timo en la etapa fetal ${ }^{19,38}$.

\section{Impacto en la comorbilidad}

Tanto el hipotiroidismo como el SOP son factores de riesgo para la presentación de múltiples alteraciones reproductivas, cardiovasculares, metabólicas y psicológicas ${ }^{48,49}$, siendo hasta el momento escasos los estudios que evalúan la presentación de estas complicaciones en pacientes con ambos trastornos.

A pesar de la falta de evidencia sobre la asociación de estas patologías, algunos autores han planteado que la presencia de hipotiroidismo podría empeorar las diferentes manifestaciones del SOP, a causa de que disminuye los niveles de globulina transportadora de hormonas sexuales, favoreciendo la conversión de androstenediona a testosterona, la aromatización a estradiol y la reducción de la depuración de androstenediona y estrona. Además, al interferir en la producción de estradiol y progesterona, estimuladas por las gonadotropinas hipofisarias, participa en la disfunción ovárica ${ }^{50}$, por lo que considerando que las mujeres con SOP presentan problemas de subfertilidad o esterilidad hasta en un $40 \%{ }^{51}$, se ha descrito que la deficiencia de hormona tiroidea y la presencia de anticuerpos antiperoxidasa (encontrados en alrededor de una cuarta parte de estas pacientes) se asocian con trastornos reproductivos y del embarazo ${ }^{6,52}$. Asimismo, se ha llegado a afirmar que el tratamiento de remplazo de 
hormona tiroidea y lograr unos valores normales de TSH en pacientes con ambas alteraciones lograría influir en la disminución de la morfología de ovarios poliquísticos y en las concentraciones séricas de andrógenos ${ }^{53,54}$.

En la actualidad, se asegura que los marcadores de riesgo cardiovascular en las pacientes con SOP son principalmente la dislipidemia y los valores elevados de homocisteína, con independencia del estado tiroide ${ }^{55}$. Sin embargo, en diferentes trabajos se ha estudiado el impacto del hipotiroidismo subclínico en el metabolismo de los lípidos y de los hidratos de carbono en mujeres con SOP ${ }^{56-63}$, encontrándose que el riesgo para enfermedades cardiovasculares puede ser mayor en las mujeres con ambas alteraciones que en aquellas con SOP y eutiroideas o sin ninguno de los dos trastornos. Esto se concluye al observar que, en las mujeres con hipotiroidismo subclínico y SOP, el colesterol total, los triglicéridos, las lipoproteínas de baja densidad, el índice HOMA para resistencia a la insulina y las mediciones de insulina tras la prueba de glucosa oral fueron significativamente más altas, en comparación con las controles, sumado a la presencia de niveles mucho más bajos de colesterol unido a lipoproteínas de alta densidad y un grosor de la íntima media de la arteria carótida significativamente menor ${ }^{62}$. Lo anterior fue corroborado por De Medeiros et al. ${ }^{64}$ en un metaanálisis, al reportar que en pacientes con SOP e hipotiroidismo subclínico se presentaron valores más altos de triglicéridos, colesterol total y glucosa en ayunas, en contraste con aquellas con SOP eutiroideas, sin demostrar adicionalmente diferencias significativas entre las medidas antropométricas, las lipoproteínas de baja densidad y los niveles de andrógenos entre ambos grupos $^{65}$. Lo anterior es concordante con el trabajo de Pergialiotis et al. ${ }^{66}$, en el que no se halló influencia del hipotiroidismo subclínico en el perfil hormonal de las mujeres con SOP, confirmando así el aumento de la gravedad de las alteraciones metabólicas del hipotiroidismo en estas pacientes y la posibilidad de un incremento de la morbilidad cardiovascular a largo plazo.

Por último, considerando la alta prevalencia de trastornos tiroideos en las mujeres con SOP, se ha planteado la necesidad de realizar un tamizaje temprano en estas pacientes, así como la corrección hacia el estado eutiroideo como una alternativa de manejo óptima para mujeres con SOP. Sin embargo, esta recomendación está limitada por la ausencia de evidencia fuerte que la respalde ${ }^{54,63}$. Del mismo modo, ante la discusión sobre la exclusión del hipotiroidismo subclínico, se ha sugerido que esta alteración no debería excluirse para establecer el diagnóstico de SOP, en ausencia de otros signos o síntomas de disfunción tiroidea ${ }^{63}$.

\section{Conclusiones}

Contemplando la importante frecuencia de enfermedad tiroidea en las mujeres con SOP y el riesgo de comorbilidad que esto implica, tanto a corto como a largo plazo, es relevante discutir la necesidad de la exclusión del hipotiroidismo de los criterios diagnósticos aplicados para el síndrome, así como la recomendación de tamizaje temprano de esta patología en las mujeres con SOP. Es necesario realizar más estudios prospectivos que evalúen las complicaciones futuras de la asociación del SOP con hipotiroidismo, las alternativas terapéuticas y los beneficios que puede traer el abordaje de ambas alteraciones en las pacientes.

\section{Financiamiento}

No hubo ningún financiamiento.

\section{Conflicto de intereses}

Los autores declaran no tener ningún conflicto de intereses.

\section{Responsabilidades éticas}

Protección de personas y animales. Los autores declaran que para esta investigación no se han realizado experimentos en seres humanos ni en animales.

Confidencialidad de los datos. Los autores declaran que han seguido los protocolos de su centro de trabajo sobre la publicación de datos de pacientes.

Derecho a la privacidad y consentimiento informado. Los autores declaran que en este artículo no aparecen datos de pacientes.

\section{Bibliografía}

1. Lizneva D, Suturina L, Walker W, Brakta S. Criteria, prevalence, and phenotypes of polycystic ovary syndrome. Fertil Steril. 2016;106:6-15.

2. March WA, Moore VM, Willson KJ, Phillips DI, Norman RJ, Davies MJ. The prevalence of polycystic ovary syndrome in a community sample assessed under contrasting diagnostic criteria. Hum Reprod. 2010;25:544-51.

3. Azziz R, Woods KS, Reyna R, Key TJ, Knochenhauer ES, Yildiz BO. The prevalence and features of the polycystic ovary syndrome in an unselected population. J Clin Endocrinol Metab. 2004;89:2745-9.

4. Azziz R, Carmina E, Dewailly D, Diamanti-Kandarakis E, Escobar-Morreale HF, Futterweit W, et al. The androgen excess and PCOS Society criteria for the polycystic ovary syndrome: the complete task force report. Fertil Steril. 2009;91:456-88.

5. Zhang HY, Zhu FF, Xiong J, Shi XB, Fu SX. Characteristics of different phenotypes of polycystic ovary syndrome based on the Rotterdam criteria in a large-scale Chinese population. BJOG. 2009;116:1633-9. 
Rev Chil Obstet Ginecol. 2021;86(5)

6. Janssen OE, Mehlmauer N, Hahn S, Offner AH, Gartner R. High prevalence of autoimmune thyroiditis in patients with polycystic ovary syndrome. Eur J Endocrinol. 2004;150:363-9.

7. Sinha U, Sinharay K, Saha S, Longkumer TA, Baul SN, Pal SK. Thyroid disorder in polycystic ovarian syndrome subjects: a tertiary hospital based cross-sectional study from Eastern India. Indian J Endocrinol Metab. 2013;17:304-9

8. Garelli S, Masiero S, Plebani M, Chen S, Furmaniak J, Armanini D, et al. Prevalence of chronic thyroiditis in patients with polycystic ovary syndrome. Eur J Obstet Gynecol. 2013;169:248-51.

9. Benetti-Pinto $\mathrm{CL}$, Berini Piccolo VRS, Garmes HM, Teatin Juliato CR. Subclinical hypothyroidism in young women with polycystic ovary syndrome: an analysis of clinical, hormonal, and metabolic parameters. Ferti Steril. 2013;99:588-92.

10. Ozdemir D, Cuhaci N, Balkan F, Usluogullari A, Ersoy R, Cakir B. Prevalence of thyroid pathologies in patients with polycystic ovary syndrome. En: $13^{\text {th }}$ Eur Cong Endocrinol. 2011; 26:92.

11. Du D, Li X. The relationship between thyroiditis and polycystic ovary syndrome: a meta-analysis. Int J Clin Exp Med. 2013;6:880-9.

12. Singla R, Gupta Y, Khemani M, Aggarwal S. Thyroid disorders and polycystic ovary syndrome: an emerging relationship. Indian J Endocrino Metab. 2015;19:25-9.

13. Vink JM, Sadrzadeh S, Lambalk CB, Boomsma DI. Heritability of polycystic ovary syndrome in a Dutch twin-family study. J Clin Endocrino Metab. 2006;91:2100-4

14. Gisel D, Carballo O, Irelys ID, Díaz S, Gilda I, Peña M, et al. Agregación familiar de causa genética en familiares de primer grado de mujeres con síndrome de ovarios poliquísticos. Rev Cuba Endocrinol. 2015;26:21-32.

15. Yildiz BO, Yarali H, Oguz H, Bayraktar M. Glucose intolerance, insulin resistance, and hyperandrogenemia in first degree relatives of women with polycystic ovary syndrome. J Clin Endocrinol Metab. 2003;88:2031-6.

16. Kahsar-Miller MD, Nixon C, Boots LR, Go RC, Azziz R. Prevalence of polycystic ovary syndrome (PCOS) in first-degree relatives of patients with PCOS. Fertil Steril. 2001:75:53-8.

17. Bothra N, Shah N, Goroshi M, Jadhav S, Padalkar S, Thakkar H, et al. Hashimoto's thyroiditis: relative recurrence risk ratio and implications for screening of first degree relatives. Clin Endocrinol (Oxf). 2017:87:201-6.

18. Akinci B, Comlekci A, Yener S, Bayraktar F, Demir T, Ozcan MA, et al. Hashimoto's thyroiditis, but not treatment of hypothyroidism, is associated with altered TGF-beta1 levels. Arch Med Res. 2008;39:397-401.

19. Gaberscek S, Zaletel K, Schwetz V, Pieber T, Obermayer-Pietsch B, Lerchbaum E. Mechanisms in endocrinology: thyroid and polycystic ovary syndrome. Eur J Endocrinol. 2015;172:R9-21.

20. Zou S, Sang Q, Wang H, Feng R, Li Q, Zhao X, et al. Common genetic variation in CYP1B1 is associated with concentrations of T4, FT3 and FT4 in the sera of polycystic ovary syndrome patients. Mol Biol Rep. 2013;40:3315-20.

21. Li Q, Yang G, Wang $Y$, Zhang X, Sang $Q$, Wang $H$, et al. Common genetic variation in the 3 '-untranslated region of gonadotropin-releasing hormone receptor regulates gene expression in cells and is associated with thyroid function, insulin secretion as well as insulin sensitivity in polycystic ovary syndrome patients. Hum Genet. 2011;129:553-61.

22. Johansson J, Stener-Victorin E. Polycystic ovary syndrome: effect and mechanisms of acupuncture for ovulation induction. Evid Based Complement Alternat Med. 2013;2013:762615

23. Cakir E, Sahin M, Topaloglu O, Colak NB, Karbek B, Gungunes A, et al. The relationship between $\mathrm{LH}$ and thyroid volume in patients with PCOS J Ovarian Res. 2012:5:43

24. Arduc A, Dogan BA, Bilmez S, Nasiroglu NI, Tuna MM, Isik S, et al. High prevalence of Hashimoto's thyroiditis in patients with polycystic ovary syndrome: does the imbalance between estradiol and progesterone play a role? Endocr Res. 2015;40:204-10.

25. Lim SS, Davies MJ, Norman RJ, Moran LJ. Overweight, obesity and central obesity in women with polycystic ovary syndrome: a systematic review and meta-analysis. Hum Reprod Update. 2012;18:618-37.

26. Rojas J, Chávez M, Olivar L, Rojas M, Morillo J, Mejías J, et al. Polycystic ovary syndrome, insulin resistance, and obesity: navigating the pathophysiologic labyrinth. Int J Reprod Med. 2014;2014:719050.

27. DeUgarte CM, Bartolucci AA, Azziz R. Prevalence of insulin resistance in the polycystic ovary syndrome using the homeostasis model assessment. Fertil Steril. 2005;83:1454-60.

28. Muscogiuri G, Sorice GP, Mezza T, Prioletta A, Lassandro AP, Pirronti T, et al. High-normal TSH values in obesity: is it insulin resistance or adipose tissue's guilt? Obesity (Silver Spring) 2013;21:101-6.

29. Song RH, Wang B, Yao QM, Li Q, Jia X, Zhang JA. The impact of obesity on thyroid autoimmunity and dysfunction: a systematic review and meta-analysis. Front Immunol. 2019;10:2349.

30. Marzullo P, Minocci A, Tagliaferri MA, Guzzaloni G, Di Blasio A, De Medici $C$, et al. Investigations of thyroid hormones and antibodies in obesity: leptin levels are associated with thyroid autoimmunity independent of bioanthropometric, hormonal, and weight-related determinants. $\mathrm{J}$ Clin Endocrinol Metab. 2010;95:3965-72.
31. Witte $\mathrm{T}$, Volzke $\mathrm{H}$, Lerch $\mathrm{M}$, Hegenscheid $\mathrm{K}$, Friedrich $\mathrm{N}$, Ittermann $\mathrm{T}$, et al. Association between serum thyroid-stimulating hormone levels and visceral adipose tissue: a population-based study in Northeast Germany. Eur Thyroid J. 2017:6:12-9.

32. Nayak PK, Mitra S, Sahoo J, Mahapatra E, Agrawal S, Lone Z. Relationship of subclinical hypothyroidism and obesity in polycystic ovarian syndrome patients. J Fam Med Prim Care. 2020;9:147-50.

33. Sahin M, Demircloglu D, Oguz A, Tuzun D, Sarica M, Inanc E. Does insulin resistance increase thyroid volume in patients with polycystic ovary syndrome? Arch Endocrinol Metab. 2017;61:145-51.

34. Wilcox G. Insulin and insulin resistance. Clin Biochem Rev. 2005:26:19-39.

35. Rotondi M, Cappelli C, Magri F, Botta R, Dionisio R, lacobello C, et al. Thyroidal effect of metformin treatment in patients with polycystic ovary syndrome. Clin Endocrinol. 2011;75:378-81.

36. Morgante G, Musachio MC, Orvieto R, Massaro MG, De Leo V. Alterations in thyroid function among the different polycystic ovary syndrome phenotypes. Gynecol Endocrinol. 2013;29:967-9

37. Marques CD, Dantas AT, Fragoso TS, Duarte AL. The importance of vitamin D levels in autoimmune diseases. Rev Bras Reumatol. 2010;50:67-80.

38. Kim D. The role of vitamin $D$ in thyroid diseases. Int $\mathrm{J}$ Mol Sci. 2017;18:1949.

39. Prietl B, Treiber G, Pieber TR, Amrein K. Vitamin D and immune function. Nutrients. 2013;5:2502-21.

40. Antico A, Tampoia M, Tozzoli R, Bizzaro N. Can supplementation with vitamin $\mathrm{D}$ reduce the risk or modify the course of autoimmune diseases? A systematic review of the literature. Autoimmun Rev. 2012;12:127-36

41. Kivity S, Agmon-levin N, Zisappl M, Shapira Y, Nagy EV, Danko K, et al. Vitamin D and autoimmune thyroid diseases. Cell Mol Immunol. 2011:8:243-7.

42. Wang J, Lv S, Chen G, Gao C, He J, Zhong H, et al. Meta-analysis of the association between vitamin $\mathrm{D}$ and autoimmune thyroid disease. Nutrients. 2015;7:2485-98.

43. He C, Lin Z, Robb SW, Ezeamama AE. Serum vitamin D levels and polycystic ovary syndrome: a systematic review and meta-analysis. Nutrients. 2015;7:4555-77.

44. Thomson RL, Spedding S, Buckley JD. Vitamin D in the aetiology and management of polycystic ovary syndrome. Clin Endocrinol (Oxf). 2012;77:343-50

45. Muscogiuri G, Palomba S, Caggiano M, Tafuri D, Colao A, Orio F. Low $25(\mathrm{OH})$ vitamin D levels are associated with autoimmune thyroid disease in polycystic ovary syndrome. Endocrine. 2016;53:538-42.

46. Fernandes $M B$, Dias $A$. Autoimmune thyroid disease in patients with polycystic ovary syndrome: the role of sexual hormones. CPQ Medicine. 2019;4:1-14

47. Chapman JC, Min SH, Freeh SM, Michael SD. The estrogen injected female mouse: new insight into the etiology of PCOS. Reprod Biol Endocrinol. 2009;7:47-57.

48. Chapman JC, Min S, Kunaporn S, Tung K, Shah S, Michael SD. The administration of cortisone to female B6A mice during their immune adaptive period causes anovulation and the formation of ovarian cysts. Am J Reprod Immunol. 2002;48:184-9.

49. Shadib SB. Subclinical hypothyroidism and its associated disorders Thyroid Disord Ther. 2018;7:2.

50. Palomba S, Santagni S, Falbo A, Battista G, Sala L. Complications and challenges associated with polycystic ovary syndrome: current perspectives. Int J Womens Health. 2015;7:745-63.

51. Wakim AN, Polizotto SL, Burholt DR. Augmentation by thyroxine of human granulose cell gonadotropin-induced steroidgenesis. Horm Reprod. 1995;10:2845-8.

52. Espinós J, Ramos A. Estudio y tratamiento de la anovulación en el síndrome de ovarios poliquísticos. Sociedad Española de Infertilidad. Guías de práctica clínica. 2020. (Citado 15/04/2020.) Disponible en: https://www.sefertilidad.net/docs/biblioteca/guiasPracticaClinicas/ guia7.pdf

53. Vissenberg R, Manders VD, Mastenbroek S, Fliers E, Afink GB, Goddijn M, et al. Pathophysiological aspects of thyroid hormone disorders/thyroid peroxidase autoantibodies and reproduction. Hum Reprod Update. 2015;21:378-87.

54. Elslimani FA, Elhasi M, Elmhdwi MF. The relation between hypothyroidism and polycystic ovary syndrome. J Pharm Appl Chem. 2016:2:197-200.

55. Fatima M, Amjad S, Sharaf Ali H Sr, Ahmed T, Khan S, Raza M, et al. Correlation of subclinical hypothyroidism with polycystic ovary syndrome (PCOS). Cureus. 2020;12:e8142.

56. Sravan Kumar $\mathrm{P}$, Ananthanarayanan $\mathrm{PH}$, Rajendiran S. Cardiovascular risk markers and thyroid status in young Indian women with polycystic ovarian syndrome: a case-control study. J Obstet Gynaecol Res. 2014;40:1361-7.

57. Huang R, Zheng J, Li S, Tao T, Liu W. Subclinical hypothyroidism in patients with polycystic ovary syndrome: distribution and its association with lipid profiles. Eur J Obstet Gynecol Reprod Biol. 2014;177:52-6.

58. Ganie MA, Laway B, Wani TA, Zargar MA, Nisar S, Ahamed F, et al. Association of subclinical hypothyroidism and phenotype, insulin resistance, and lipid parameters in young women with polycystic ovary syndrome. Fertil Steril. 2011;95:2039-43. 
59. Benetti-Pinto CL, Berini Piccolo VR, Garmes HM, Teatin Juliato CR. Subclinical hypothyroidism in young women with polycystic ovary syndrome: an analysis of clinical, hormonal, and metabolic parameters. Fertil Steril. 2013;99:588-92.

60. Dittrich R, Kajaia N, Cupisti S, Hoffman I, Beckmann MW, Mueller A. Association of thyroid-stimulating hormone with insulin resistance and androgen parameters in women with PCOS. Reprod Biomed Online. 2009; 19:319-25

61. Celik C, Abali R, Tasdemir N, Guzel S, Yuksel A, Aksu E, et al. Is subclinical hypothyroidism contributing dyslipidemia and insulin resistance in women with polycystic ovary syndrome? Gynecol Endocrinol. 2012;28:615-8.

62. Enzevaei A, Salehpour S, Tohidi M, Saharkhiz N. Subclinical hypothyroidism and insulin resistance in polycystic ovary syndrome: is there a relationship? Iran J Reprod Med. 2014;12:481-6.
63. Pei YJ, Wang AM, Zhao Y, Yan L, Li M, White RE, et al. Studies of cardiovascular risk factors in polycystic ovary syndrome patients combined with subclinical hypothyroidism. Gynecol Endocrinol. 2014;30:553-6.

64. De Medeiros SF, De Medeiros MS, Ormond CM, Barbosa JS, Yamamoto MM. Subclinical hypothyroidism impact on the characteristics of patients with polycystic ovary syndrome. A meta-analysis of observational studies. Gynecol Obstet Invest. 2018:83:105-15.

65. De Medeiros SF, Yamamoto MM, De Medeiros MS, Barbosa JS, Norman RJ. Should subclinical hypothyroidism be an exclusion criterion for the diagnosis of polycystic ovary syndrome? J Reprod Infertil. 2017;18:242-50.

66. Pergialiotis V Konstantopoulos P, Prodromidou A Florou V Papantoniou N, Perrea DN. Management of endocrine disease: the impact of subclinical hypothyroidism on anthropometric characteristics, lipid, glucose and hormonal profile of PCOS patients: a systematic review and meta-analysis. Eur J Endocrinol. 2017;176:159-66. 